\title{
ESTIMATIVAS DO TEOR DE UMIDADE EMPREGANDO 0 MÉTODO GPR: UMA AVALIAÇÃO COMPARATIVA EM EXPERIMENTOS DE LABORATÓRIO E CAMPO
}

\author{
Emílio Eduardo Moreira Barbosa ${ }^{1}$, Renato Luiz Prado², Rodolfo Moreda Mendes 3 \\ e Fernando Antônio Medeiros Marinho ${ }^{4}$ \\ Recebido em 6 novembro, 2009 / Aceito em 13 outubro, 2010 \\ Received on November 6, 2009 / Accepted on October 13, 2010
}

\begin{abstract}
This paper makes an analysis of the quantitative correspondence between the GPR-TDR and GPR-gravimetric measurements of moisture content. The data were obtained from laboratory and field experiments. In the laboratory test, the vadose zone was simulated by injecting and draining water in a sand tank. The experiment was conducted with $1 \mathrm{GHz}$ antennas. The in situ test was carried out on unsaturated residual soils, in an area susceptible to landslide, with $200 \mathrm{MHz}$ antennas. To measure soil water content with GPR, we used the velocity of ground wave, refracted and reflected waves. The GPR response was sensitive to changes in subsurface moisture in both experiments and showed good correspondence with TDR (RMS error of $0.007 \mathrm{~m}^{3} \mathrm{~m}^{-3}$ ) and gravimetric (RMS error of $0.039 \mathrm{~m}^{3} \mathrm{~m}^{-3}$ ) measurements.
\end{abstract}

Keywords: GPR, TDR, moisture content, dielectric constant.

RESUMO. Este trabalho faz uma análise das estimativas de teores de umidade obtidas com o método Ground Penetrating Radar (GPR) comparativamente às determinadas com os métodos Time Domain Reflectometry (TDR) e gravimétrico. Os dados foram obtidos em dois experimentos diferentes: um experimento controlado em laboratório buscando reproduzir um meio homogêneo onde foram obtidas as medidas de umidade com GPR (antenas de $1 \mathrm{GHz}$ ) e TDR, e outro experimento de campo onde foram obtidos dados com GPR (antenas de $200 \mathrm{MHz}$ ) e de amostras de solos do local. Para a obtenção das estimativas a partir do método GPR foram analisados os eventos relativos à onda de transmissão direta entre as antenas, onda refratada criticamente e onda refletida em interfaces com diferentes propriedades elétricas. 0 GPR mostrou-se sensível às variações de umidades presentes nos dois experimentos e apresentou boa correlação com os dados obtidos com TDR (REQM de $0,007 \mathrm{~m}^{3} \mathrm{~m}^{-3}$ ) e das amostras (REQM de $0,039 \mathrm{~m}^{3} \mathrm{~m}^{-3}$ ).

Palavras-chave: GPR, TDR, teor de umidade, constante dielétrica.

\footnotetext{
1 Programa de Pós-graduação, Instituto de Astronomia, Geofísica, e Ciências Atmosféricas, Universidade de São Paulo, Rua do Matão, 1226, Cidade Universitária, 05508-090 São Paulo, SP, Brasil. Tel.: (11) 3091-2789; Fax: (11) 3091-5094 - E-mail: emilio@iag.usp.br

2 Instituto de Astronomia, Geofísica e Ciências Atmosféricas, Universidade de São Paulo, Rua do Matão, 1226, Cidade Universitária, 05508-090 São Paulo, SP, Brasil. Tel.: (11) 3091-2762; Fax: (11) 3091-5034 - E-mail: renato@iag.usp.br

${ }^{3}$ Instituto Geológico do Estado de São Paulo, Avenida Miguel Stéfano, 3900, Água Funda, 04301-903 São Paulo, SP, Brasil. Caixa-Postal: 8772. Tel.: (11) 5058-9994 (Ramal 2047); Fax: (11) 5073-5511 - E-mail: rodolfommendes@ig.com.br

${ }^{4}$ Escola Politécnica, Universidade de São Paulo, Avenida Professor Almeida Prado, 271, Cidade Universitária, 05508-900 São Paulo, SP, Brasil. Tel.: (11) 3091-5703; Fax: (11) 3818-5181-E-mail: fmarinho@usp.br
} 


\section{INTRODUÇÃo}

A medida do teor de umidade de solos é importante em diversas áreas de aplicação como na engenharia geotécnica e engenharia agrícola, em estudos de estabilidade de encostas e regimes de irrigação. Empregando os métodos Ground Penetrating Radar (GPR) ou Time Domain Reflectomery (TDR) 0 teor de umidade pode ser obtido indiretamente a partir da determinação da permissividade dielétrica do meio e de relações empíricas como a obtida por Topp et al. (1980). A despeito das diferenças existentes entre os dois métodos (a medida TDR é pontual uma vez que traduz a condição de umidade existente ao redor da sonda, e a medida GPR representa a "média" de um volume por onde transitou a frente de onda) suas medidas são frequentemente comparadas para se avaliar a acurácia do valor obtido com o GPR (Huisman et al., 2002; Galagedara et al., 2003).

Este trabalho busca avaliar a estimativa de umidade pelo método GPR a partir da análise dos resultados obtidos em duas situações distintas de levantamento: i) um experimento controlado em laboratório buscando reproduzir um meio isotrópico onde foram obtidas medidas de umidade com GPR e TDR; ii) um levantamento de campo (solos de alteração de granitos) onde os resultados do GPR foram confrontados com os obtidos de amostras deformadas coletadas com trado manual.

Para a obtenção dessas estimativas foram analisados todos os eventos presentes nos radargramas: onda de transmissão direta entre as antenas, onda refratada criticamente e onda refletida em interfaces com diferentes propriedades elétricas. Cada um desses eventos representa diferentes profundidades/volumes de amostragem e variações na acurácia das estimativas.

\section{MATERIAIS E MÉTODOS}

\section{Experimento de laboratório}

Antes da montagem do experimento foram realizados estudos de modelagem com 0 software ReflexW (Sandmeier, 2006) variando-se os parâmetros físicos do meio (condutividade elétrica e constante dielétrica considerando o meio seco e saturado) e as posições dos discos metálicos que seriam inseridos em diferentes profundidades. Como o experimento seria montado com elementos disponíveis no laboratório (antena GPR, tanque, sonda TDR, areia, discos metálicos), as modelagens visaram essencialmente à análise qualitativa dos dados sintéticos nos aspectos relacionados às eventuais interferências entre as hipérboles de difração geradas pelos discos e à atenuação do sinal.

A Figura 1 mostra 0 resultado da modelagem 2D referente ao meio "seco" simulando uma aquisição common offset para a disposição de discos que posteriormente foi adotada no experimento. 0 radargrama sintético gerado indica a possibilidade de identificação de reflexões oriundas de discos posicionados até a profundidade de $1,8 \mathrm{~m}$.

Na montagem do experimento empregou-se: tanque cilíndrico de fibra de vidro de 2,1 $\mathrm{m}$ de altura e 1,4 m de diâmetro preenchido com areia fina selecionada e dotado de sistema de injeção e drenagem de água na sua base; discos metálicos de 0,13 m de diâmetro e 0,5 cm de espessura; bem como sondas TDR.

A Figura 2 apresenta esquema ilustrativo da disposição dos discos metálicos e pares de TDRs no tanque e da linha L1 de aquisição GPR, assim como uma fotografia do tanque com detalhe do sistema inferior de enchimento/drenagem. Os pares de sensores TDR foram posicionados em níveis intercalados aos dos discos metálicos, um oposto ao outro, e contidos em um plano transversal ao dos discos.

Os dados foram adquiridos no modo transversal elétrico (TE) ao longo da linha de disposição dos discos com uma antena biestática blindada de $1 \mathrm{GHz}$ de frequência. A escolha da antena esteve diretamente relacionada à área da seção do tanque disponível para a realização do ensaio. Como parâmetros de aquisição foram adotados: espaçamento entre traços de $2 \mathrm{~cm}$; intervalo de amostragem de 0,077 ns; 1024 amostras e empilhamento de 128 traços.

0 experimento consistiu na simulação da zona vadosa em duas condições distintas de umidade. Assim, foram analisados os radargramas adquiridos antes da injeção do fluido no tanque, e imediatamente após a drenagem. Desta forma foram simuladas diferentes condições de umidade (aqui denominadas "seca" e pós-drenagem) uma vez que processos de capilaridade e adsorção permitem a retenção de pequena parte do volume injetado.

Concomitante às aquisições com GPR foram feitas leituras com os TDRs a cada 5 minutos durante todo o período do experimento (antes do início do enchimento, durante 0 enchimento e a drenagem). Essas medidas são apresentadas na Figura 3. A medida TDR é baseada no tempo de trânsito do pulso eletromagnético ao longo de uma haste metálica inserida no meio investigado.

Nos radargramas foram identificadas as hipérboles de difração geradas pela presença dos discos metálicos. A partir delas foram obtidas as velocidades médias dos "pacotes" situados acima dos níveis dos discos através do ajuste semiautomático das hipérboles feito com o aplicativo ReflexW.

Visando à comparação com as constantes dielétricas dos TDRs, converteu-se as velocidades $(V)$ obtidas do dado GPR 
A)

\begin{tabular}{ccc}
\hline Parâmetros & areia & disco metálico \\
\hline $\mathrm{K}$ & 6,5 & 300 \\
\hline$\sigma(\mathrm{mS} / \mathrm{m})$ & 0,01 & 99,99 \\
\hline$\mu_{\mathrm{r}}$ & 1 & 100 \\
\hline
\end{tabular}

B)

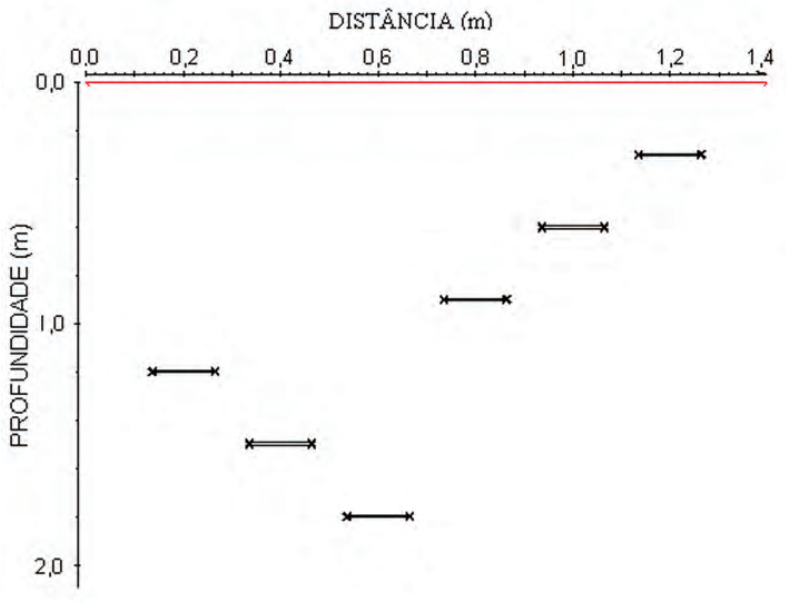

C)

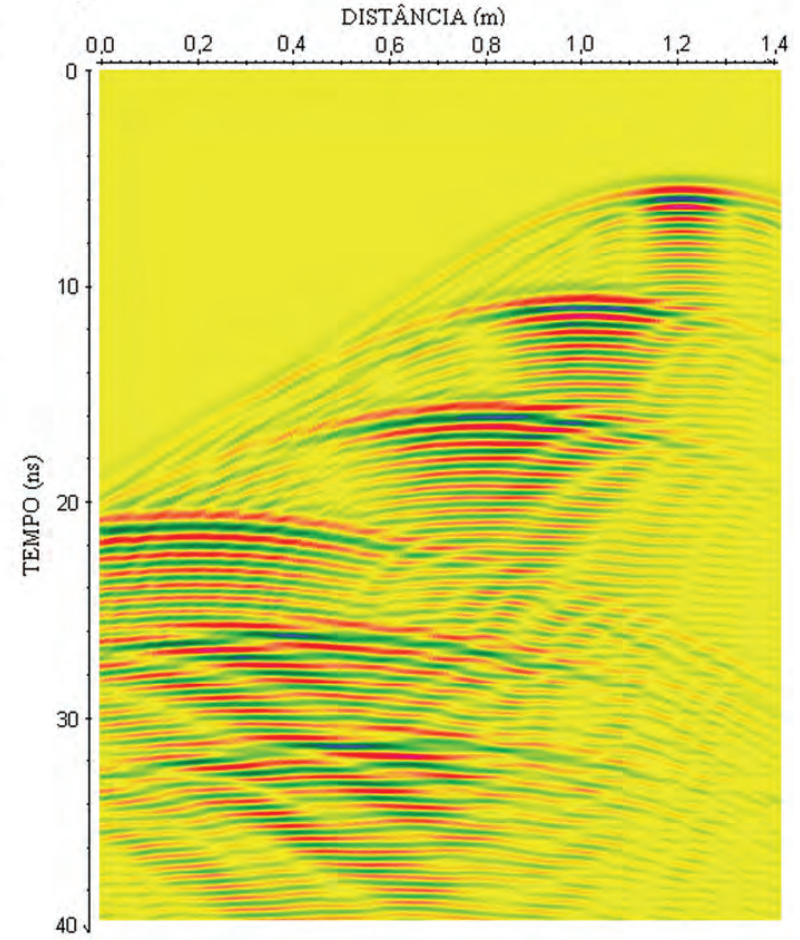

Figura 1 - Radargrama sintético referente ao meio "seco" gerado pelo método das diferenças finitas (ReflexW). (A) Parâmetros do modelo: constante dielétrica (K); condutividade elétrica $(\sigma)$ e permeabilidade magnética relativa $\left(\mu_{r}\right)$. (B) Geometria do modelo com indicação dos retângulos representando os discos metálicos e (C) radargrama sintético obtido com esse modelo (frequência principal do sinal simulado =1 GHz).

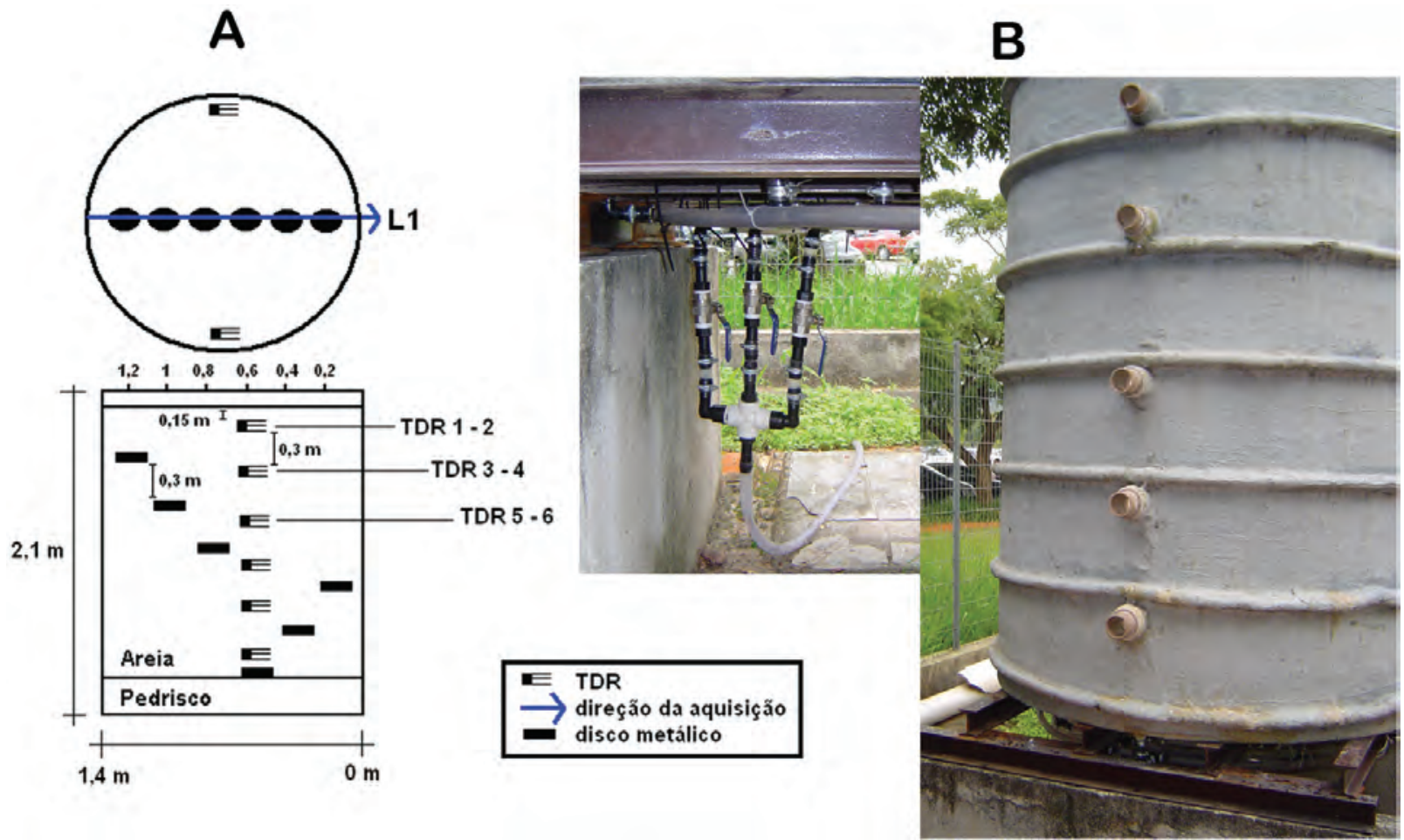

Figura 2 - A) Representação esquemática da disposição dos discos metálicos e pares TDRs no tanque com areia e da linha L1 de aquisição GPR. B) Fotografia do tanque de realização dos ensaios com detalhe do sistema inferior de enchimento/drenagem. 


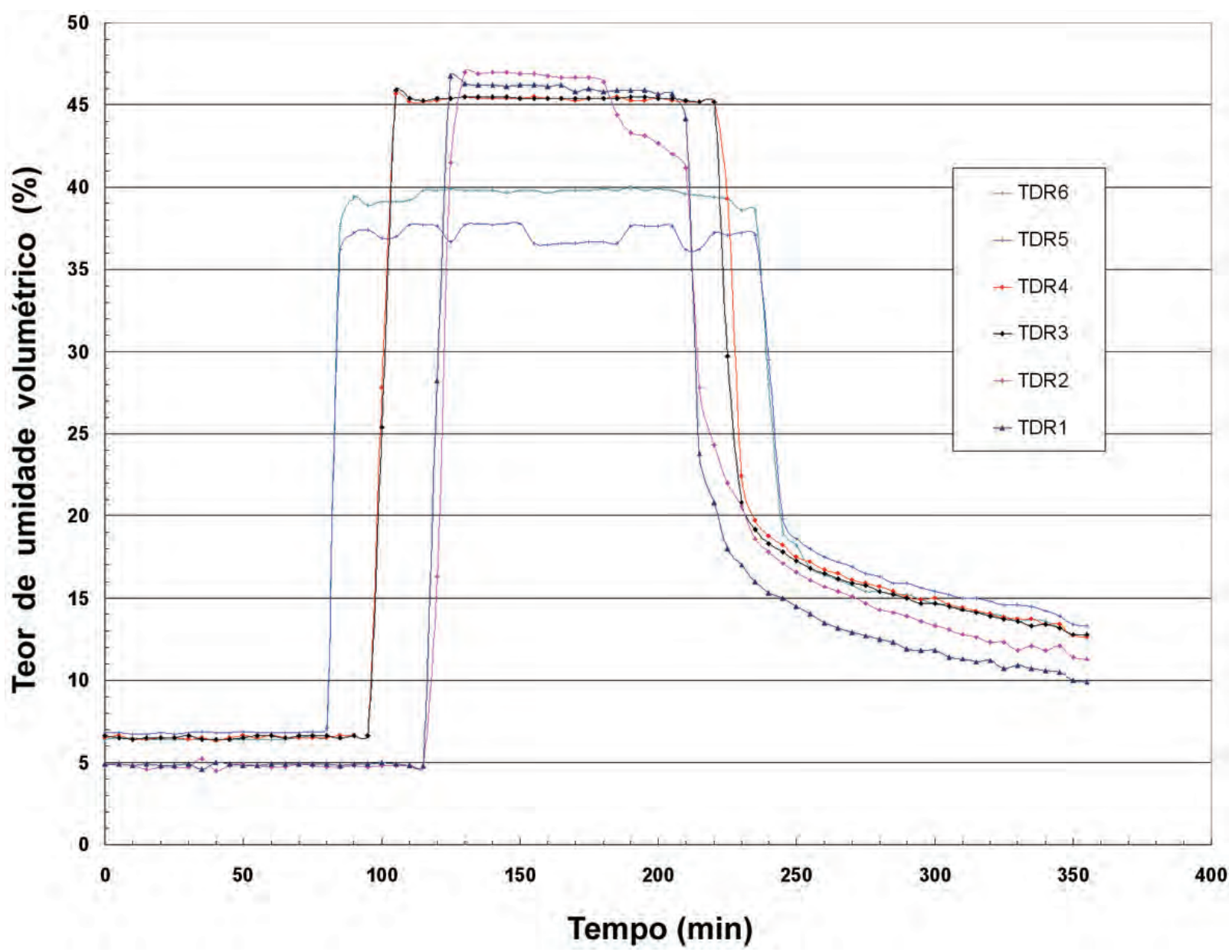

Figura 3 - Dados de constante dielétrica obtidos das sondas TDRs de 1 a 6 (em ordem de profundidade) em função do tempo (leituras a cada 5 minutos).

para valores de constante dielétrica $(K)$ a partir da Equação (1), onde $c$ é a velocidade da onda eletromagnética $(E M)$ no vácuo. A equação é válida para as altas frequências empregadas nas aquisições GPR e para meios de baixa perda (pouco condutivos):

$$
K=\left(\frac{c}{V}\right)^{2}
$$

Embora a estimativa do conteúdo volumétrico de água pelo TDR também possa conter erros devido aos vazios eventualmente existentes entre 0 solo e as sondas (Ferre et al., 1998; Sakaki et al., 1998), e também devido às incertezas na técnica de medida do tempo de percurso usada pelo instrumento, seus resultados nesta comparação foram tomados como referência, uma vez que 0 uso da técnica TDR é de plena aceitação nas áreas da engenharia geotécnica e agrícola.

Nesta comparação considerou-se como valor representativo do TDR em cada nível a média das leituras obtidas pelo respectivo par de sensores. Por se tratar de meio poroso, isotrópico e homogêneo, se esperava pequenas variações da velocidade da onda EM ao longo do perfil vertical, motivadas pela contínua diminuição do volume de poros com a profundidade em razão do peso dos sedimentos.

Como os valores obtidos das sondas TDR traduziam condições de umidade do local de posição do sensor, enquanto os do GPR o da média de um volume de material contido da superfície até um respectivo disco, foram feitas duas comparações: uma entre os valores representando o volume entre discos adjacentes (referidas como valores "intervalares"), e outra entre os valores médios do "pacote" (da superfície até o disco), para cada método.

No primeiro caso (valores "intervalares"), obteve-se $K$ dos radargramas após o cálculo das velocidades intervalares (Dix, 1955) e $K$ dos TDRs pela média aritmética dos valores obtidos nos dois sensores instalados no correspondente intervalo de profundidade. 
No segundo caso (valores do "pacote"), foi calculada uma média dos valores das constantes dielétricas dos TDRs ponderada em relação às espessuras $h_{i}$ (intervalo de profundidade entre os discos):

$$
K_{M}=\frac{\sum_{i=1}^{n} K_{i} h_{i}}{\sum_{i=1}^{n} h_{i}}
$$

Para a estimativa dos teores de umidade foi empregada a proposição de Topp et al. (1980) que estabelece uma relação empírica entre a constante dielétrica, $K$, e 0 teor de umidade volumétrico, $\theta\left(\mathrm{m}^{3} \mathrm{~m}^{-3}\right)$ :

$$
\begin{aligned}
\theta= & -5,3 \times 10^{-2}+2,92 \times 10^{-2} K \\
& -5,5 \times 10^{-4} K^{2}+4,2 \times 10^{-6} K^{3}
\end{aligned}
$$

\section{Experimento de campo}

As aquisições dos dados foram efetuadas numa área do município de Ubatuba, Estado de São Paulo, local onde foram realizados estudos de caracterização de solos residuais sujeitos a escorregamentos (Ferreira, 2004; Mendes, 2008). Sua localização é apresentada na Figura 4.

Utilizou-se o sistema RAMAC da Mala Geosciences e antenas biestáticas não blindadas de $200 \mathrm{MHz}$. 0 modo de aquisição empregado foi 0 common midpoint (CMP) com afastamento mínimo de $0 \mathrm{~m}$, máximo de $12 \mathrm{~m}$ e intervalos de aquisição de $0,1 \mathrm{~m}$.

Para a comparação com os dados GPR também foram obtidas medidas "pontuais" de teor de umidade volumétrico a partir de amostras deformadas de solo coletadas com um trado mecânico em seguida à aquisição GPR. 0 furo de trado foi feito próximo $(\approx 0,10 \mathrm{~m}$ ) à posição central do arranjo CMP com a coleta de amostras a cada $0,25 \mathrm{~m}$, até a profundidade máxima de $3,0 \mathrm{~m}$.

As amostras foram hermeticamente embaladas, assim que coletadas. No laboratório foram pesadas e em seguida secadas em uma estufa a $105^{\circ} \mathrm{C}$. Já secas foram novamente pesadas e assim obteve-se a massa de água pela diferença das pesagens. 0 teor de umidade gravimétrico $(w)$ foi obtido pela razão entre a massa de água e a massa da amostra seca. Os teores de umidade volumétricos $(\theta)$ foram obtidos com a Equaçã̃o (4) onde $\rho_{d}$ representa a massa específica aparente seca. Os valores de $\rho_{d}$ foram obtidos de amostras indeformadas (Mendes, 2008).

$$
\theta=w \cdot \rho_{d}
$$

Após o processamento dos dados GPR (correção tempo ze- ro, filtro passa-banda e ganho a partir da curva média de decaimento da amplitude), foi feita a análise de velocidades dos eventos da onda direta e refratada (ajuste linear) e das ondas refletidas nos diferentes horizontes do solo de alteração. As velocidades das ondas refletidas foram obtidas através de ajustes hiperbólicos e análise dos máximos da função semblance no espectro de velocidades (Yilmaz, 1987).

A permissividade dielétrica foi obtida com a Equação (1) e as estimativas dos teores de umidade com a Equação (3).

0 teor de umidade volumétrico calculado através do evento da onda direta traduz a propriedade do solo até uma profundidade específica $\left(d_{i}\right)$, chamada de profundidade de influência. Neste trabalho empregamos a proposta de van Overmeeren et al. (1997) para o cálculo de $d_{i}$, dada por:

$$
d_{i}=\frac{1}{2} \sqrt{\frac{V S}{f}}
$$

onde $V$ é a velocidade de propagação da onda direta, $S$ a distância entre antenas e $f$ a frequência dominante do sinal.

Para o cálculo das profundidades $(d)$ dos pacotes determinados pelas hipérboles de reflexão identificadas nos radargramas, utilizou-se a Equação (6), onde $V_{N M O}$ é a velocidade média obtida diretamente da análise de velocidades (melhor correção normal moveout) e $t_{o}$ o respectivo tempo duplo de percurso para 0 afastamento nulo.

$$
d=\frac{V_{N M O} \cdot t_{o}}{2}
$$

\section{RESULTADOS E DISCUSSÕES \\ Experimento de laboratório}

A Figura 5 apresenta os radargramas obtidos no experimento controlado (meios "seco" e pós-drenagem). Embora a energia do sinal eletromagnético tenha sido suficiente para registrar o sinal refletido dos discos mais profundos, como previsto nos estudos de modelagem (Fig. 1), há interferências das hipérboles superiores, o que contribui para um aumento da incerteza nas medidas das velocidades. Deste modo, optou-se pela análise somente das três primeiras reflexões indicadas na Figura 5 .

A Figura 6 apresenta a comparação GPR/TDR das estimativas dos teores de umidade feita através de regressão linear simples considerando os diferentes critérios (valores intervalares e valores dos "pacotes"). A Tabela 1 apresenta as raízes dos erros quadráticos médios (REQM) entre os valores de $\theta$ (teor de umidade volumétrico) obtidos do GPR e dos sensores TDR para cada comparação, em cada meio, além dos coeficientes angular, linear e de determinação $\left(R^{2}\right)$ de cada ajuste linear. 

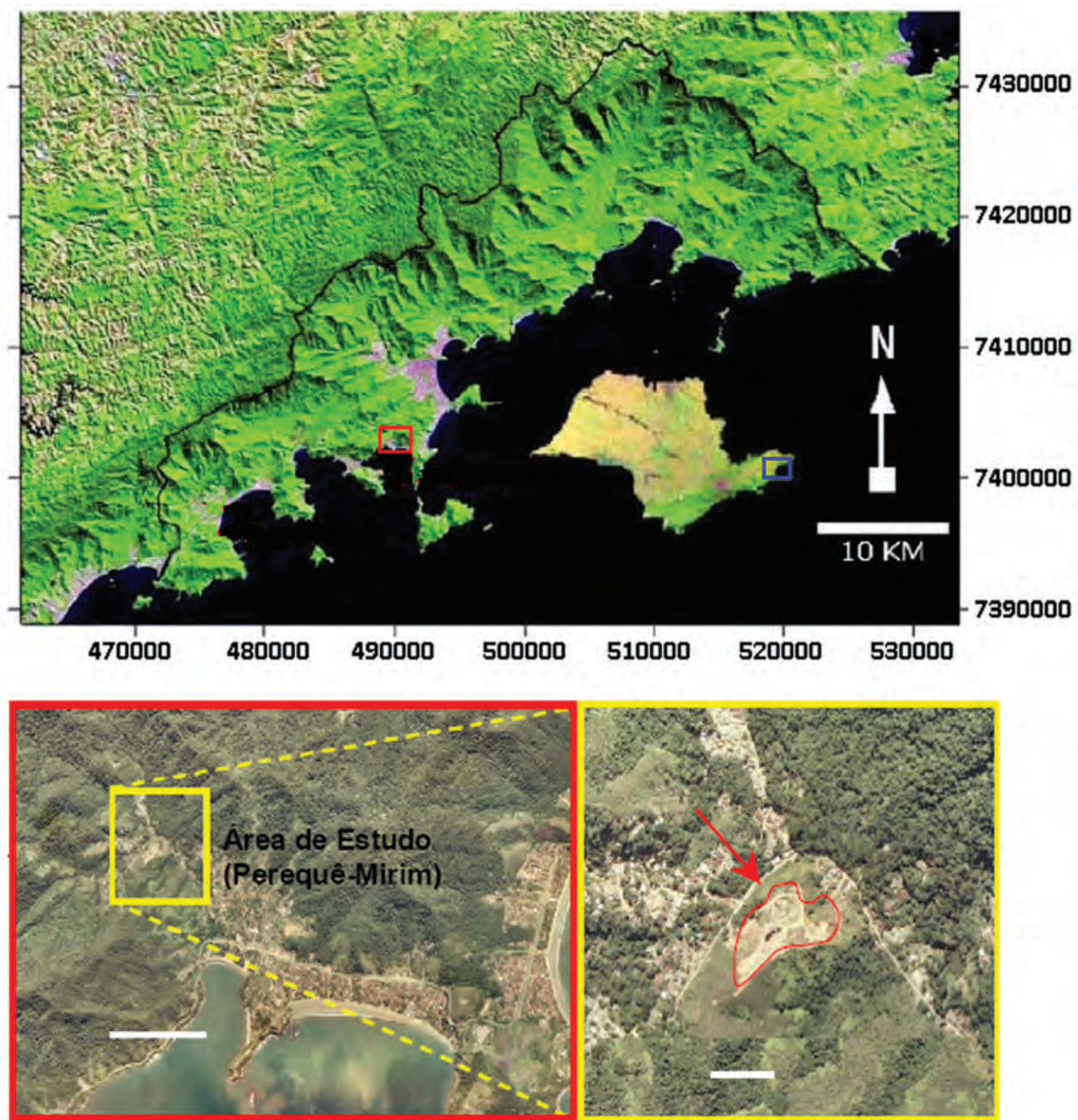

Figura 4 - Localização da área de estudo no município de Ubatuba. Projeção UTM, datum Córrego Alegre (adaptado de Mendes, 2008).

Observa-se que a resposta GPR foi sensível às diferentes condições de umidade do meio (do "seco" para o drenado). No entanto, os valores obtidos das velocidades intervalares foram os menos precisos, como se constata da Figura 6 e da REQM da Tabela 1. Atribui-se este fato à aplicação da fórmula de Dix (Dix, 1955) aos dados GPR quando as diferenças de velocidades e tempos de percurso entre reflexões sucessivas são pequenas (levando-se em conta que sempre haverá erros associados às suas determinações, quaisquer que sejam os métodos empregados na análise de velocidades). Tal situação também é passivel de ocorrer em levantamentos GPR de campo em investigações rasas de meios estratificados

Os valores médios (obtidos das velocidades NMO) foram os que apresentaram as menores REQMs (Tab. 1). Em se tratando da zona vadosa, onde não se espera variações abruptas de umidade, 0 uso de valores médios pode traduzir a condição de umidade do meio. 0 baixo valor da REQM obtido indica boa precisão na medida da umidade volumétrica com o método GPR, tendo-se como referência as leituras com TDR.

Independente do teor de umidade do meio, uma maior razão entre sinal e ruído no dado GPR resultará em uma estimativa mais precisa. 

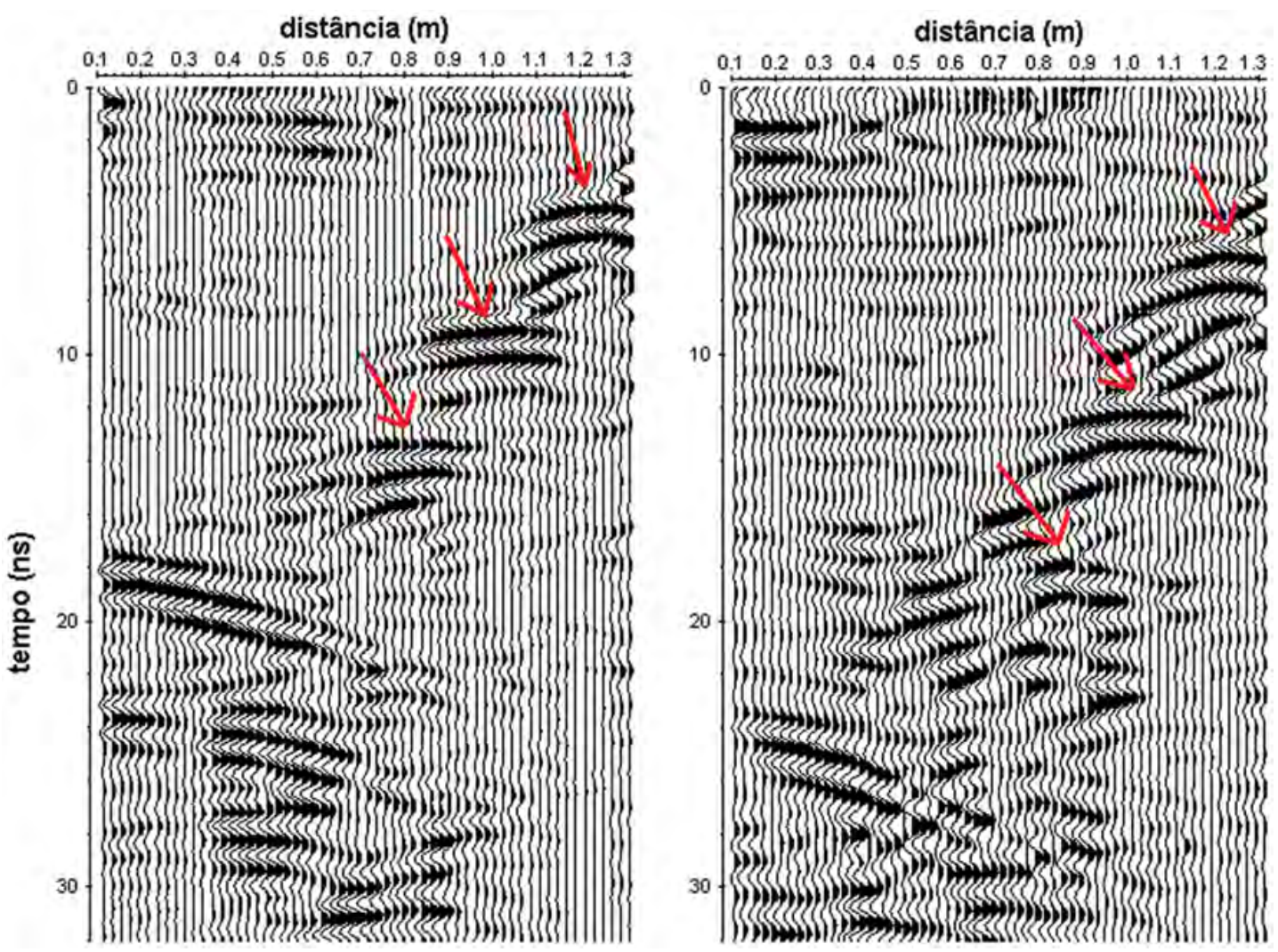

Figura $\mathbf{5}$ - Radargramas referentes às aquisições nos meios "seco" e após a drenagem. As setas indicam as 3 primeiras hipérboles de cada seção escolhidas para análise neste trabalho.

Tabela 1 - Valores dos parâmetros dos ajustes lineares e REQM de cada comparação em cada meio.

\begin{tabular}{|c|c|c|c|c|c|}
\hline Meio & Comparação & Coef. angular & Coef. linear & $\mathrm{R}^{2}$ & $\mathrm{REQM}\left(\mathrm{m}^{3} \mathrm{~m}^{-3}\right)$ \\
\hline \multirow{2}{*}{ Seco e drenado } & intervalar & 1,01 & 0,68 & 0,76 & 0,026 \\
\cline { 2 - 6 } & "pacotes" & 0,90 & 0,76 & 0,98 & 0,007 \\
\hline
\end{tabular}

$\mathrm{R}^{2}$ : Coeficiente de determinação; REQM: Raiz do erro quadrático médio.

\section{Experimento de campo}

A análise de velocidades dos dados do experimento de campo também foi feita com base no ajuste de hipérboles diretamente nos radargramas, assim como pelo espectro de velocidades da função semblance. A partir da verificação, no experimento controlado, das incertezas associadas ao cálculo das velocidades intervalares, optou-se, no caso do experimento de campo, somente pelo emprego das velocidades NMO para a obtenção dos valores de $K$ e umidade.

A Figura 7 mostra o radargrama CMP e seu respectivo espectro de velocidade com a indicação dos máximos de coerência locais.
0 gráfico da Figura 8 apresenta os valores de umidade volumétrica obtidos dos dados GPR (onda direta, refratada e refletida) e das amostras de campo. Os erros das medidas de umidade e profundidades obtidos do evento reflexão foram estimados a partir das abrangências dos máximos da função semblance nos espectros de velocidades (domínio $t_{0}-V_{N M O}$ ).

Os dados obtidos das amostras de solo (Fig. 8) mostram que ocorre uma diminuição contínua de umidade até a profundidade aproximada de 1,4 $\mathrm{m}$, e a partir desta um pequeno aumento até os $3 \mathrm{~m}$.

Também da interpretação dos eventos no radargrama obtido no ensaio CMP, pode-se observar a ocorrência de refração crítica motivada pelo fato de haver uma maior umidade em um hori- 


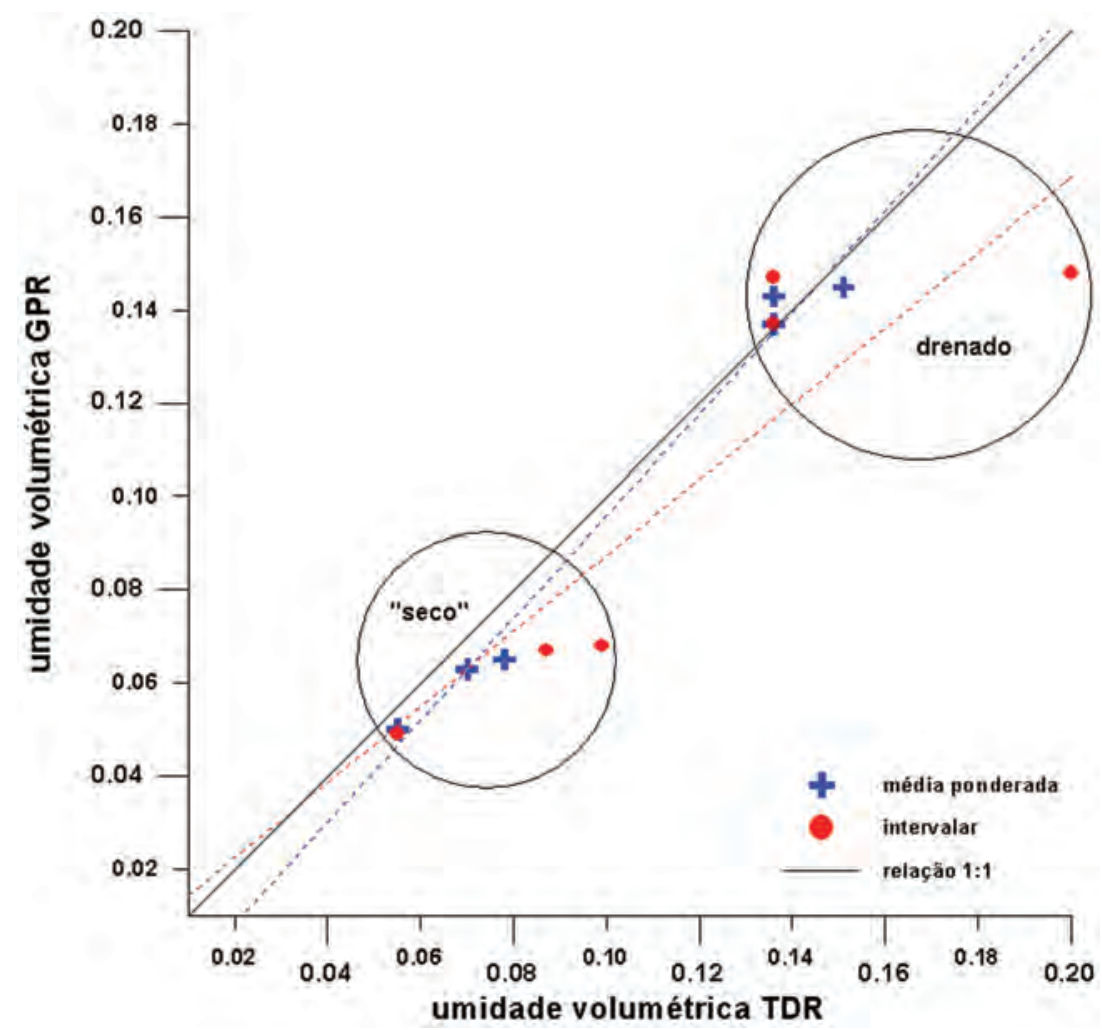

Figura 6 - Comparação entre os valores $K_{G P R}$ e $K_{T D R}$ considerando os valores intervalares e valores médios dos "pacotes" para o meio "seco" e drenado.

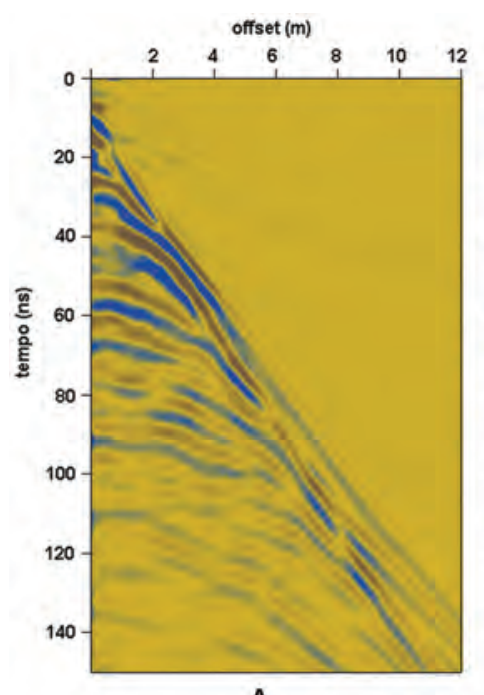

A

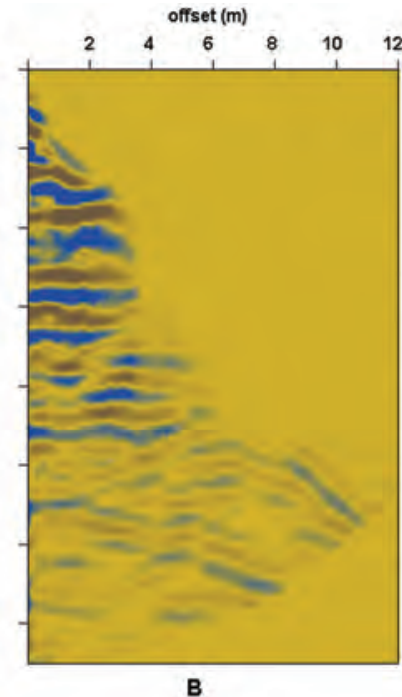

B
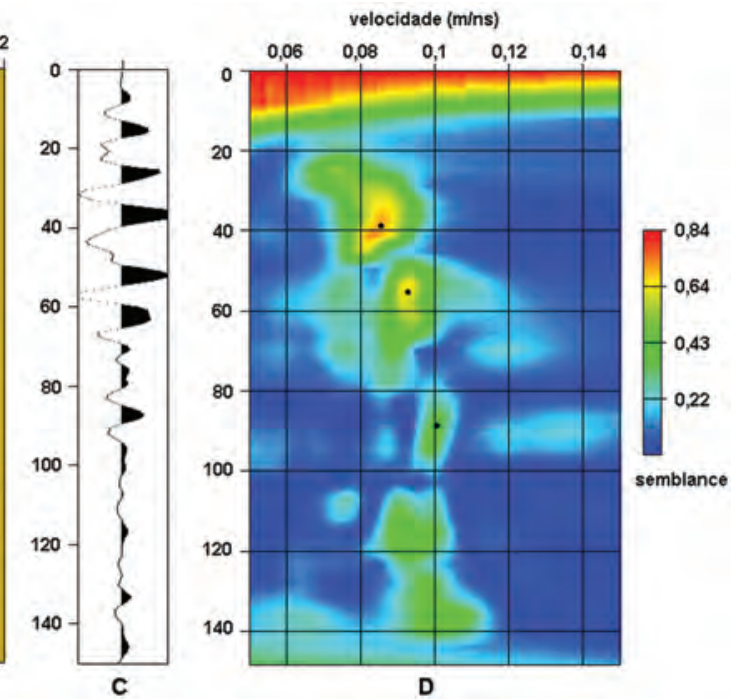

Figura 7 - Exemplo de resultado de análise de velocidades do dado do experimento de campo pelo método semblance: A) radargrama processado; B) radargrama após correção NMO; C) traço empilhado; D) espectro de velocidade com indicação dos máximos de coerência locais.

zonte de solo mais raso, ou seja, nesse horizonte a velocidade média de propagação da onda EM é menor (Fig. 9). A profundidade dessa interface, calculada pelo método da refração, foi de
1,45 m. A profundidade de influência do percurso da onda direta, calculada com a Equação (5) e considerando offset máximo de $1,8 \mathrm{~m}$, foi de $0,6 \mathrm{~m}$. 


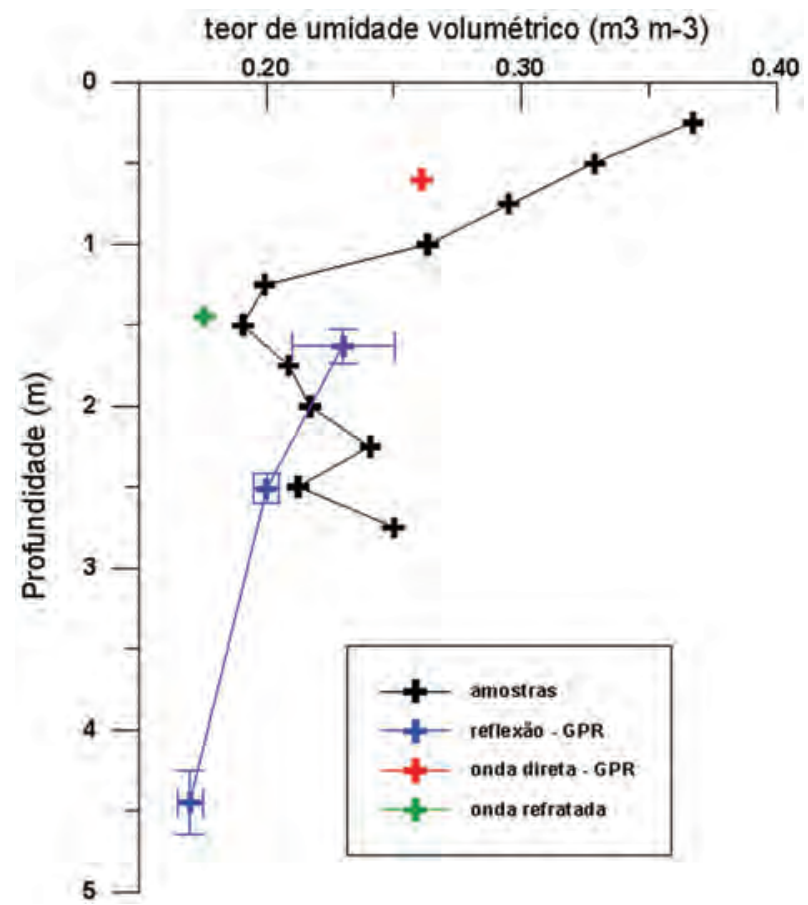

Figura 8 - Teores de umidade volumétricos obtidos dos dados GPR e das amostras de campo plotados com suas respectivas profundidades.

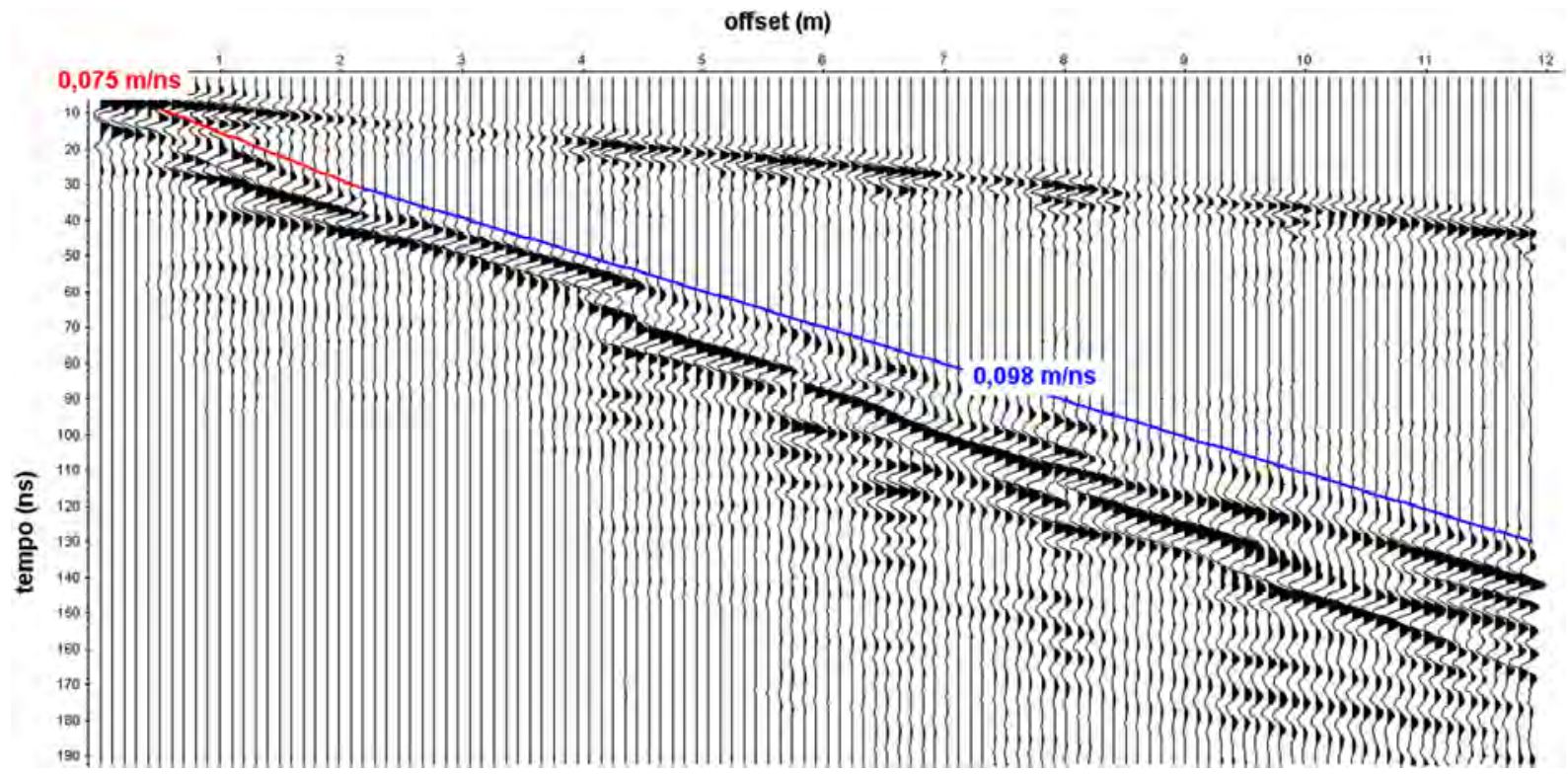

Figura 9 - Radargrama com destaque para os eventos referentes à onda direta $(V=0,075 \mathrm{~m} / \mathrm{ns})$ e onda refratada $(V=0,098 \mathrm{~m} / \mathrm{ns})$.

No caso do evento reflexão as profundidades foram estimadas a partir de $t_{0}$ (tempo duplo da trajetória normal) e $V_{N M O}$ (obtida da análise de velocidade).

Assim como no experimento controlado, foram comparados os dados obtidos com o GPR, representativos de médias de um pacote de solo (considerando o evento reflexão), com os valores "pontuais" obtidos das amostras em cada nível de coleta. Desta forma, foi necessária a obtenção de uma média dos valores das amostras de solo para possibilitar a comparação direta com os dados GPR. 


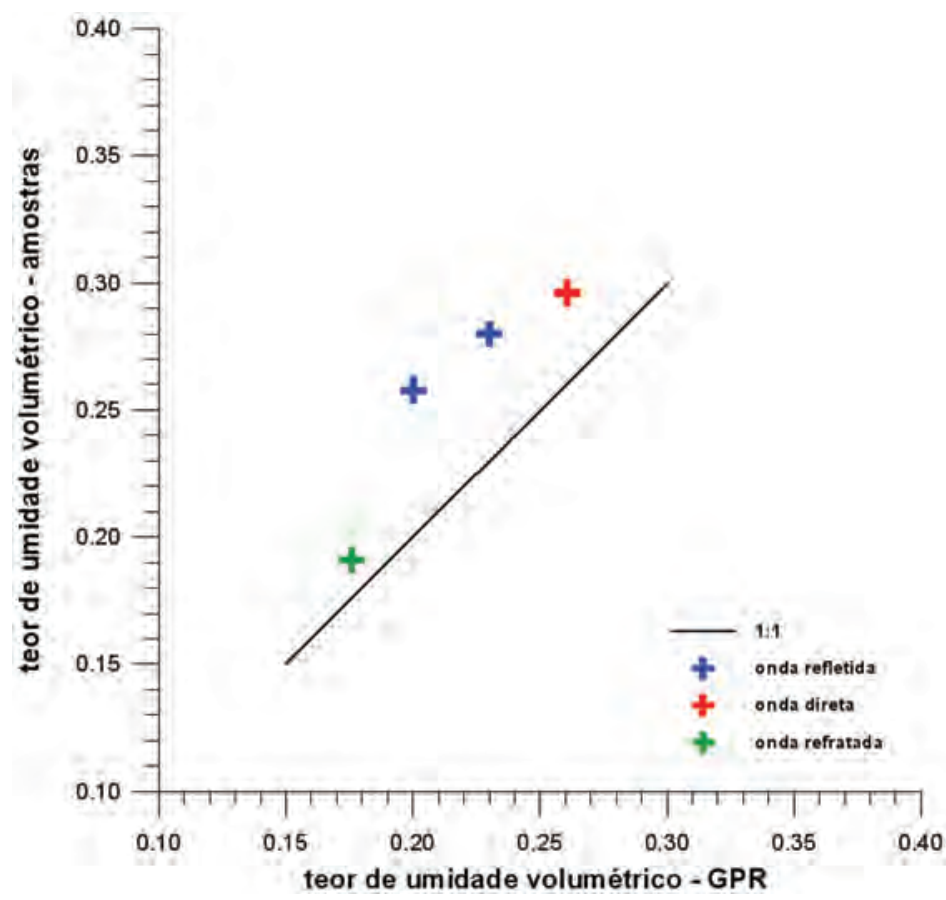

Figura 10 - Análise comparativa entre os teores de umidade obtidos com o GPR e com as médias $\left(\theta_{m}\right)$ obtidas com os valores pontuais das amostras de solo.

0 estudo controlado feito em laboratório mostrou que uma melhor correlação entre valores médios de GPR e valores "pontuais" ocorre quando a média estabelecida para estes últimos é ponderada com suas correspondentes profundidades. Desse modo, utilizou-se a expressão da Equação (7) para estimar os valores médios de teor de umidade volumétrico $\left(\theta_{m}\right)$ obtidos das amostras para diferentes profundidades $\left(d_{n}\right)$ :

$$
\theta_{m}=\left(\frac{\sum_{i=1}^{n}\left[\theta_{i}^{2} \cdot\left(d_{i}-d_{i-1}\right)\right]}{d_{n}}\right)^{0,5}
$$

onde $n$ é 0 número de amostras até a qual se quer obter $\theta_{m}$ para o pacote correspondente, $i$ é o número da amostra, e $\theta_{i}$ e $d_{i}$ são respectivamente a umidade e a profundidade da iésima amostra.

Como a profundidade máxima de coleta de amostras foi de $3 \mathrm{~m}$, pôde-se somente analisar comparativamente as umidades obtidas das duas primeiras reflexões observadas no dado GPR, ou seja, relativas às interfaces situadas nas profundidades de $1,63 \pm 0,21 \mathrm{~m}$ e 2,51 $\pm 0,18 \mathrm{~m}$. A comparação é apresentada nas Figuras 8 e 10. Pode-se observar que os valores de umidade obtidos dos dados GPR foram próximos aos dos valores das amostras, embora sistematicamente inferiores para quaisquer dos eventos analisados. A REQM da regressão linear da Fi- gura 10 foi de $0,039 \mathrm{~m}^{3} \mathrm{~m}^{-3}$, valores comparáveis aos obtidos por Huisman et al. (2001).

\section{CONCLUSÕES}

0 ensaio de laboratório mostrou que o método GPR foi bastante sensível às variações das condições de umidade do meio mesmo considerando somente 0 evento de reflexão de onda, fornecendo medidas precisas de umidade até as profundidades onde 0 sinal apresentou boa relação sinal/ruído.

Os resultados de laboratório também mostraram que se deve dar preferência ao cálculo de teores de umidade obtidos a partir das velocidades médias (NMO) dos eventos de reflexão, uma vez que os cálculos dos valores intervalares resultam em maiores erros em se tratando de dados GPR.

Situações distintas de teor de umidade (pré-enchimento e pós-drenagem no ensaio de laboratório) apresentaram a mesma precisão.

Mesmo no ensaio em ambiente natural, onde as condições são mais distantes das ideais, o que normalmente resulta em baixa relação sinal-ruído, obteve-se boa precisão na medida da umidade (REQM 0,039 $\mathrm{m}^{3} \mathrm{~m}^{-3}$ ). Neste caso considerando os três diferentes eventos analisados (onda direta, refratada e refletida). A separação por eventos permite ainda a investigação em diferentes escalas de profundidade. 
As limitações associadas às investigações diretas, TDR (invasivo, pontual, erros na presença de macroporos, canais de raízes e agregados) e amostragem de solos (invasivo, pontual, erros em solos expansivos), e os baixos erros observados neste estudo para as medidas GPR, apontam este método como uma ferramenta importante para avaliações da variação espacial (em diferentes escalas de profundidade) e temporal dos teores de umidade da subsuperfície rasa.

\section{AGRADECIMENTOS}

Os autores agradecem à FAPESP (auxílio pesquisa 05/55736-5), ao IAG-USP e à EP-USP pelo apoio laboratorial, ao Prof. Orlando M. Oliveira do DEC-UFSC pela colaboração na concepção e montagem do experimento, e aos revisores pelas correções e sugestões.

\section{REFERÊNCIAS}

DIX CH. 1955. Seismic velocities from surface measurements. Geophysics, 20: 68-86.

FERRE PA, KNIGHT JH, RUDOLPH DL \& KACHANOSKI RG. 1998. The Sample areas of conventional and alternative time domain reflectometry probes. Water Resource Research, 34(11): 2971-2979.

FERREIRA CJ. 2004. Diretrizes para a regeneração sócio-ambiental de áreas degradadas por mineração de saibro, Ubatuba (SP). Relatório Técnico (Processo Fapesp 03/07182-5). 50 p.

GALAGEDARA LW, PARKIN GW, REDMAN JD \& ENDRES AL. 2003. Assessment of soil moisture content measured by borehole GPR and TDR under transient irrigation and drainage. Journal of Engineering and Environmental Geophysics, 8(2): 26-35.
HUISMAN JA, SPERL C, BOUTEN W \& VERSTRATEN JM. 2001. Soil water content measurements at different scales: accuracy of time domain reflectometry and ground-penetrating radar. Journal of Hydrology, 245: $48-58$.

HUISMAN JA, SNEPVANGERS JJJC, BOUTEN W \& HEUVELINK GBM. 2002. Mapping spatial variation in surface soil water content: comparison of ground-penetrating radar and time domain reflectometry. Journal of Hydrology, 269: 194-207.

MENDES RM. 2008. Estudo das propriedades geotécnicas de solos residuais não saturados de Ubatuba (SP). Tese (Doutorado em Engenharia), Escola Politécnica, Universidade de São Paulo, São Paulo, 236 p.

SAKAKI T, SUGIHARA K, ADACHI T, NISHIDA K \& LIN W. 1998. Application of time domain reflectometry to determination of volumetric water content in rock. Water Resources Research, 24(10): 2623-2631.

SANDMEIER KJ. 2006. ReflexW Version 3.5.8. Program for the processing of seismic, acoustic or electromagnetic reflection, refraction and transmission data.

TOPP GC, DAVIS JL \& ANNAN AP. 1980. Electromagnetic determination of soil water content measurements in coaxial transmission lines. Water Resources Research, 16(3): 574-582.

VAN OVERMEEREN RA, SARIOWAN SV \& GEHRELS JC. 1997. Ground penetrating radar for determining volumetric soil water content; results of comparative measurements at two test sites. Journal of Hydrology, 197: 316-338.

YILMAZ 0. 1987. Seismic Data Processing. Tulsa: Society of Exploration Geophysicists, 538 p.

\section{NOTAS SOBRE OS AUTORES}

Emílio Eduardo Moreira Barbosa. Bacharel em Geofísica (IAG/USP, 2009). Atualmente é pós-graduando em Geofísica pelo IAG-USP.

Renato Luiz Prado. Bacharel em Geologia (IGc/USP, 1981), mestre em Geofísica (IAG/USP, 1994) e doutor em Geociências e Meio Ambiente (IGCE/UNESP, 2000). Professor do IAG da Universidade de São Paulo.

Rodolfo Moreda Mendes. Engenheiro Civil pela FEIS-UNESP - Ilha Solteira (1997), mestre em Engenharia Urbana (UFSCar, 2001) e doutor em Engenharia Geotécnica (EP/USP, 2008). Pesquisador científico do Instituto Geológico do Estado de São Paulo.

Fernando Antônio Medeiros Marinho. Engenheiro Civil pela Universidade de Pernambuco (1983), mestre em Mecânica dos Solos (PUC-RJ, 1986), doutor em Geotecnia pela University of London (1994) e pós-doutorado pela Arizona State University (2008). Professor associado da EP da Universidade de São Paulo. 